\title{
Fast Evaluation of Multi-Hadron Correlation
} Functions

\section{Pranjal Vachaspati*}

Massachusetts Institute of Technology $y^{\dagger}$

E-mail: vachasp2eillinois.edu

\section{William Detmold}

Massachusetts Institute of Technology

E-mail: wdetmold@mit.edu

\begin{abstract}
Calculating the values of nuclear correlation functions is computationally intensive due to the fact that the number of terms in a nuclear wave function scales exponentially with atomic number. To speed up this computation, we represent a correlation function as a sum of the determinants of many small matrices, and exploit similarities between the matrices to speed up the calculations of those determinants.
\end{abstract}

The 32nd International Symposium on Lattice Field Theory,

23-28 June, 2014

Columbia University New York, NY

\footnotetext{
* Speaker.

$\dagger$ Currently at the University of Illinois at Urbana-Champaign
} 


\section{Introduction}

We wish to find the correlation function between a source and a sink nuclear wave function, of the form

$$
\left\langle\mathscr{N}_{1}(t) \mid \mathscr{N}_{2}(0)\right\rangle
$$

where $\overline{\mathscr{T}}_{h}$ and $\mathscr{N}_{h}$ are nuclear creation and annihilation operators built from quark and gluon fields described by quantum numbers $h$. As described in [1], $\overline{\mathscr{N}}_{h}$ can be written as

$$
\overline{\mathscr{N}}_{h}=\sum_{\{a\}} w_{h}^{a_{1} \ldots a_{n_{q}}} \bar{q}\left(a_{1}\right) \bar{q}\left(a_{2}\right) \ldots \bar{q}\left(a_{n_{q}}\right)
$$

where $w_{h}$ is an antisymmetric tensor that is nonzero only when none of the indices are equal and the combination of the quarks is appropriate for the nuclear quantum number.

The correlation function requires a sum over pairs of terms in the source and sink wavefunctions, and each pair requires a sum over orderings of quark creation and annihilation operators in each term. It can be expressed in terms of quark propagators $S\left(q\left(a_{0}\right), q\left(a_{1}\right) ; t\right)$ as:

$$
\left\langle\mathscr{N}_{1}(t) \mid \overline{\mathscr{N}}_{2}(0)\right\rangle=\frac{1}{Z} \int d U e^{-S_{Q C D}} \sum_{i \in s r C} \sum_{j \in s n k} w_{i} w_{j} \sum_{\alpha, \beta} S\left(\bar{q}\left(a_{i}^{\alpha_{1}}\right), q\left(a_{j}^{\beta_{1}}\right)\right) \ldots S\left(\bar{q}\left(a_{i}^{\alpha_{n q}}\right), q\left(a_{j}{ }^{\beta_{n q}}\right)\right) \varepsilon^{\alpha} \varepsilon^{\beta}
$$

where $\alpha$ and $\beta$ are permutations of $1 \ldots n q$, and $a_{n}^{m}$ is the $m^{\text {th }}$ creation or annihilation operator in the $n^{\text {th }}$ term of the wavefunction.

For a given source term $i$ and sink term $j$, the sum over $\alpha$ and $\beta$ is equivalent to taking the determinant of a matrix $G^{(i j)}$ where [1]

$$
G_{m n}^{(i j)}=S\left(\bar{q}\left(a_{i}^{m}\right), q\left(a_{j}^{n}\right)\right)
$$

Therefore, the expression for the correlator can be restated as:

$$
\left\langle\mathscr{N}_{1}(t) \mid \overline{\mathscr{N}}_{2}(0)\right\rangle=\frac{1}{Z} \int d U e^{-S_{Q C D}} \sum_{i \in \text { srC }} \sum_{j \in \text { snk }} w_{i} w_{j} \operatorname{det}\left(G^{(i j)}\right)
$$

Here, computing the nuclear correlation function requires computing a determinant for every pair of terms in the sink and source wave functions, which typically grow rapidly in size with increasing atomic number $A$. Note that $G$ is block-diagonal because the correlation function between two quarks of different flavors is always zero, so we compute $\operatorname{det}(G)$ as the product of the determinants of smaller single-flavor matrices.

\section{Determinant Updates}

Typically, $\operatorname{det}(G)$ is calculated by taking the trace of the $L U$ decomposition of the matrix, which takes $O\left(n^{3}\right)$ time in the number of quarks [2]. However, this can be improved by taking into account similarities between pairs of matrices.

If two source wavefunctions or two sink wavefunctions differ by only one term, the corresponding $G$ matrices will differ by only a single row or column. If source terms $i$ and $j$ differ only 
in the $r^{\text {th }}$ element, the matrices $G^{(i k)}$ and $G^{(j k)}$, which denote, respectively, the correlator between the $i^{\text {th }}$ or $j^{\text {th }}$ source term and the $k^{\text {th }}$ sink term, can be expressed as:

$$
G^{(j k)}=G^{(i k)}+u v^{T}
$$

where $v$ is the difference between the rows of $G^{(j k)}$ and $G^{(i k)}$, and $u$ is an element vector that determines which row has changed.

\subsection{Matrix Determinant Lemma}

An update of this form can be computed in $O\left(n^{2}\right)$ time, using the identity [3]

$$
\operatorname{det}\left(G+u v^{T}\right)=\left(1+v^{T} G^{-1} u\right) \operatorname{det}(G) .
$$

To prove this, we first see that:

$$
\operatorname{det}\left(I+u v^{T}\right)=\left(1+v^{T} u\right)
$$

because of the equality

$$
\left(\begin{array}{cc}
1 & 0 \\
v^{T} & 1
\end{array}\right)\left(\begin{array}{cc}
1+u v^{T} & u \\
0 & 1
\end{array}\right)\left(\begin{array}{cc}
1 & 0 \\
-v^{T} & 1
\end{array}\right)=\left(\begin{array}{cc}
I & u \\
0 & 1+v^{T} u
\end{array}\right)
$$

This implies

$$
\operatorname{det}\left(G+u v^{T}\right)=\operatorname{det}(G) \operatorname{det}\left(I+G^{-1} u v^{T}\right)=\operatorname{det}(G) \operatorname{det}\left(I+v^{T} G^{-1} u\right)
$$

$G^{-1} u$ is easily computed using the $L U$ factorization, since the systems $U x=b$ and $L y=x$ can be solved in a total of $n^{2}-n$ multiply-adds and $2 n$ divides.

Somewhat surprisingly, it is possible to calculate some rank-1 updates in $O(n)$ time. If a parent matrix $G^{(i k)}$ has the $n^{\text {th }}$ row changed in one way to get $G^{(j k)}$ and in a different way to get $G^{(l k)}$, the determinant of $G^{(j k)}$ is

$$
\operatorname{det}\left(G^{(i k)}+u v_{1}^{T}\right)=\left(1+v_{1}^{T} G^{(i k)^{-1}} u\right) \operatorname{det}\left(G^{(i k)}\right),
$$

and the determinant of $G^{l k}$ will be

$$
\operatorname{det}\left(G^{(i k)}+u v_{2}^{T}\right)=\left(1+v_{2}^{T} G^{(i k)^{-1}} u\right) \operatorname{det}\left(G^{(i k)}\right) .
$$

The most computationally intensive calculation is finding $G^{(i k)^{-1}} u$, but this is shared between the two determinants. So the determinant of $G^{(l k)}$ can be computed with only a single vector multiply.

\section{Execution Order}

To be able to use the fast determinant method, we must find a set $S$ of matrices such that the every matrix $G$ differs from some matrix in $S$ by at most one row or column. Then, after evaluating $\operatorname{det}\left(G_{1}\right)$ where $G_{1} \in S$, which takes $O\left(n^{3}\right)$ time, all the matrices that differ from $G_{1}$ by a row or column can have their determinants calculated in $O\left(n^{2}\right)$ or $O(n)$ time. 


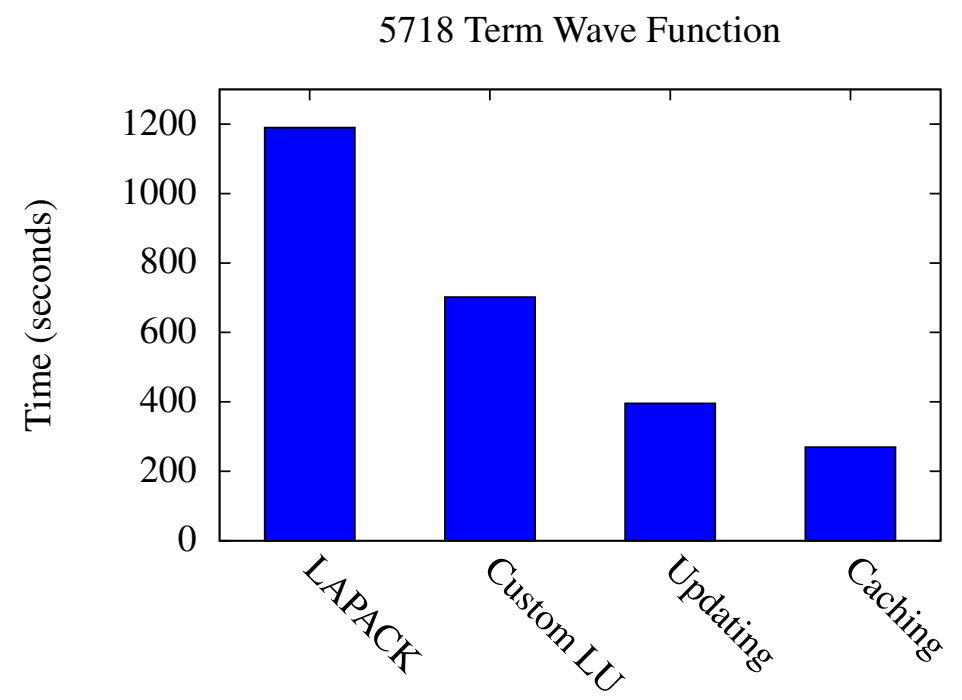

Figure 1: A comparison of algorithms shows that the fast calculation is approximately four times as fast as LAPACK. "Updating" uses the $O\left(n^{2}\right)$ update algorithm where it can, and "caching" additionally saves the solution of $G^{-1} u$ to compute a portion of the determinants in $O(n)$ time.

Consider a graph in which each matrix is a vertex and an edge exists between vertices if they differ by a single row or column. The subset $S$ is represented by a dominating set of this graph. Ideally, we would use the minimal dominating set, as this would require the fewest uses of the $O\left(n^{3}\right)$ algorithm.

However, finding the minimal dominating set of a graph is known to be $N P$-complete. Luckily, a simple greedy algorithm, where the vertex with the highest degree is chosen and removed from the graph, along with its neighbors, provides a good approximation [4].

Furthermore, simply generating the graph is difficult, as $O\left(N^{4}\right)$ matrix comparisons are needed. For this reason, only the dominating set of the source wavefunction is considered. This has complexity of only $O\left(N^{2}\right)$, which takes no more than a few minutes given a wave function with order $10^{4}$ terms. The graph and dominating set remain the same over different gauge configurations, so the time spent in this phase can be amortized over many correlator calculations.

In practice, the vertices in the graph tend to have high degree, so a large fraction of the matrices can have their determinants calculated in linear or quadratic time.

\section{Results}

This method was tested using five sample alpha particle wave functions. One had all 12 quarks on the same site with one term; two consisted of a triton and a proton, with 1944 terms each; and two had two particles on each site, with 5718 terms each. All combinations of wavefunctions at the sink and source were considered, so when both the sink and source had 5718 terms, a total of $5718^{2}=32,695,524$ determinants were calculated per site.

The wave function with one term had a dominating set of size one; the 1944 term wave functions had dominating sets of size $167,183,241$, and 245 ; and the 5718 term wave functions had 
dominating sets of sizes 509, 568, 699, and 703. Thus, between 80 and 90 percent of the determinants could be calculated more quickly than with the naïve method.

First, the LU factorization function in LAPACK was replaced with a custom method that did not use pivoting, a technique that enhances numerical stability for poorly conditioned matrices. LAPACK is optimized for larger matrices; for the small $(6 \times 6)$ matrices used here, LAPACK has considerable overhead. The optimizations discussed above were applied using this custom factorization function.

Overall, the determinant updating technique was between four and five times faster than LAPACK when tested on combinations of the wave functions above, as can be seen in Figure 1.

\section{Conclusion \& Further Improvements}

Improving two portions of the algorithm would have significant impacts on efficiency.

The first is the quality of the dominating set approximation, which determines how many determinants must be computed in cubic time. A simple greedy algorithm works reasonably well, but it may be worthwhile to spend more time in this phase to get a better result. Second, reducing overhead in the algorithm and in the caching mechanism becomes increasingly important as the asymptotic complexity of the determinant calculation decreases.

Larger nuclei would benefit more from this approach, since the difference between the $O\left(n^{3}\right)$ approach and the faster methods would be more significant. For sufficiently large nuclei, utilizing rank-2 and higher updates might be effective as well.

Additionally, this method can be parallelized up to the size of the dominating set, as the graph can be partitioned into sets which each contain one member of the dominating set and its neighbors. Each of these partitions can be considered separately.

Utilizing the similarities between wavefunction terms can provide significant speedups in the calculation of correlators. The matrix determinant lemma provides an asymptotic speedup from $O\left(n^{3}\right)$ to $O\left(n^{2}\right)$ for many of the terms, and careful caching provides a further speedup to $O(n)$. This asymptotic speedup is reflected in real-world correlator calculations.

\section{References}

[1] William Detmold and Kostas Orginos. Nuclear correlation functions in lattice qcd. Physical Review D, 87(11):114512, 2013.

[2] Gene H Golub and Charles F Van Loan. Matrix computations, volume 3. JHU Press, 2012.

[3] Jiu Ding and Aihui Zhou. Eigenvalues of rank-one updated matrices with some applications. Applied Mathematics Letters, 20(12):1223-1226, 2007.

[4] Abhay K Parekh. Analysis of a greedy heuristic for finding small dominating sets in graphs. Information processing letters, 39(5):237-240, 1991. 